\title{
An New Approach for Acceleration of Train Model with Compressed Air
}

\author{
Q.S. Yang ${ }^{1}$, J.H. Song ${ }^{1,2}$, and Guowei Yang ${ }^{1}$ \\ ${ }^{1}$ Laboratory of High Temperature Gas Dynamics, Institute of Mechanics, \\ Chinese Academy of Sciences, Beijing 100190, China \\ ${ }^{2}$ Research Institute of Photonics, Dalian Polytechnic University, Dalian 116034, China
}

\begin{abstract}
In order to accelerate a heavy train model with a large dimension to a velocity higher than $500 \mathrm{~km} /$ hour for the moving model test, the compressed air is utilized to drive the model indirectly. The gas from an airgun impels a piston in the accelerating tube for a uniformly acceleration. The piston connects a driving device through a rope and the device promotes or pulls the train model for a desired velocity. The deceleration of the train model and the driving device are all realized by the friction between the brake plates and the rubber blocks. The principium experiment results reveal that the initial pressure of the airgun is linearly proportional to the kinetic energy of the model where the moving assembly with a weight more than $10 \mathrm{~kg}$ has been accelerated to $420 \mathrm{~km} / \mathrm{hour}$ in an accelerating distance of $20 \mathrm{~m}$.
\end{abstract}

Keywords: Moving train model experiments, Airgun, Compressed air, Friction Deceleration.

\section{Introduction}

In order to investigate the aerodynamic performs of a train configuration with a high velocity, it is required to accelerate a heavy model to a defined velocity within a limited distance in the moving model testing system where the model is with a large dimension. Having been measured in the testing section, the moving model enters the deceleration section, begins to move with a deceleration, and finally stops. The difficulty of the acceleration and deceleration of the model as well as the corresponding expenditure increases with the weight of the model and the acceleration of model are essential for a successful moving model testing system.

Recently, one of the methods is the utilization of the elastic rope for the kinetic energy of the train model [1]. A set of traveling pulley is connected to the rope and the dragline of the train model is twisted on the wheels of the pulley. Thus, the velocity of the model can be increased into several times of that of the elastic rope and the ratio of the velocity depends on the structure of the pulley. This technology is suited to the acceleration of the model with a large mass and the experiments on such test system can be performed for the experimental simulation 
of the aerodynamics of the train configuration. However, it is disadvantageous to accelerate the train model with a velocity higher than $300 \mathrm{~km} / \mathrm{hour}$ due to a great friction between all the wheels and the towrope since the rotation of the wheels can not provide a line speed matching the moving towrope. For the purpose of the experimental simulation of the moving train in People's Republic of China, the train model should be faster than $350 \mathrm{~km} /$ hour and as high as $500 \sim 600 \mathrm{~km} /$ hour.

Another type for accelerating a train model is the utilization of the compressed air where the train model is driven by the compressed air in a tube[2-5]. Although the model can be accelerated to a velocity higher than $500 \mathrm{~km} /$ hour, this technology is unsuited to the launching of the model with a larger dimension due to the following reasons. Firstly, the cross dimension and the configuration of the model is limited by the inner size of the launching tubes since the model is gliding during the acceleration of the model. The dimension of the train model is proportional to both the inner size of the launching tube and, therefore, the cost of the experimental device. Secondly, the installation of the braking device of the model is also restricted by the launching tube where it is required for the model to be decelerated and finally to be stopped after it has passed though the experiment section. On the other hand, it is very difficult to stop a matching piston, which is used to push the model and seal the compressed air, at the accelerating end of the launching tube as the aerodynamic configuration of the train is experimental simulated. Fourthly, the compressed air flowing from the launching tube will seriously disturb the experimental measurement for the aerodynamic characteristics of the train profile. Although the flowing can be partially eliminated by a large cavity device [5], this technique can not be utilized in the experimental device where the two models move at high speeds

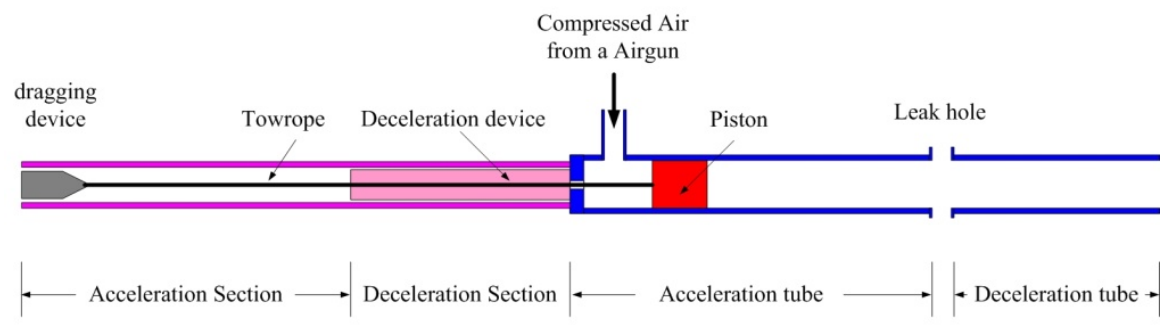

( a )

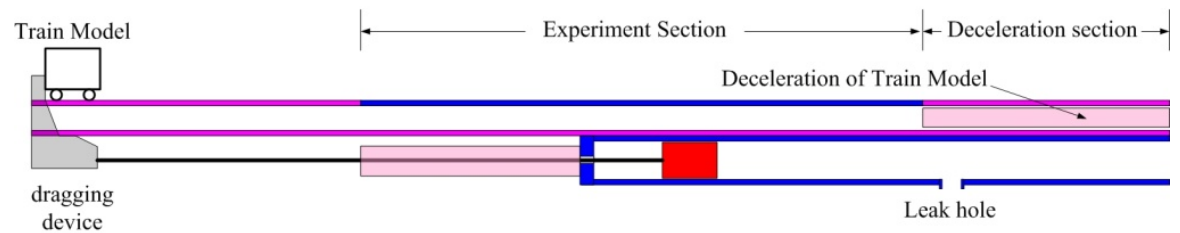

(b)

Fig. 1 Structure sketch of the acceleration of the train model (a) the acceleration of the piston and the dragging device by compressed air in the bottom and (b) Side view of the acceleration part 
with two opposite directions in two parallel tracks and the tracks are with a limited vertical distance. Finally, the pantograph model can not be installed at the top of the train model due to the launching tube and, therefore, the experimental simulation of the train model with the pantograph can not be carried out if the model is launched from a launching tube.

In order to develop a moving train model testing system, which will be used to accelerate a train model with a heavy mass and large dimension to a higher velocity than $500 \mathrm{~km} /$ hour, for the experimental simulation of a series of train model, a project has been established where the total length of the device and the maximum of the train model are $180 \mathrm{~m}$ and $40 \mathrm{~kg}$, respectively and the power of accelerating the model is from the compressed air. In the testing system, the model with a pantograph can be resembled to the aerodynamic configuration of the trains.

\section{Acceleration of Train Model by Compressed Air}

The sketch of the device on the acceleration of the train model by the compressed air is shown in Figure 1. In the acceleration tube, the compressed air from an airgun drives a piston and the latter connects to a dragging device, which slides on a set of double track. The towrope between the piston and the dragging device passes through a hole in the closed end of the acceleration tube, as shown in Figure 1 (a). And this part locates at the bottom structure of the device.

In the top, the model undergoes three processes: acceleration, testing, and deceleration, as shown in Figure 1(b). The leakage of the compressed air from the hole can be ignored because the diameter of the hole is much smaller than that of the acceleration tube. The dragging device draws the train model, which will slide on another set of double track. As the piston reaches another end of the acceleration tube, the acceleration process of the piston, the dragging device, and the train model finishes due to the leakage of the compressed air through the leak hole. Then, the dragging device begin to slow due to the existence of the deceleration segment of the dragging device and also makes the piston slow down because of the towrope. Due to the inertia, the model continues to moving forward and into the testing section.

In the development of the moving train model testing system, in order to to validate the principle and the technical feasibility of the project above and to determine the operating parameters of the system, the relevant experimental investigations are carried out.

\section{Experimental Validation of the Technique and the Result Analysis}

The first kind of experiment is the acceleration of piston by compressed air, as shown in Figure 2. The compressed air from the air-gun enters the room between the piston and closed end of the acceleration tube and pushes the piston move in the right direction. After the piston passes through the leak hole, the compressed air escapes out from the acceleration tube and the acceleration process ends. The 
velocity measurement is finished by the sheltering of laser beam by the piston, which is recorded by an oscillograph. The diameter and the length of the acceleration tube are $110 \mathrm{~mm}$ and $1500 \mathrm{~mm}$, respectively. The weights of two pistons are $1.7 \mathrm{~kg}$ and $3.3 \mathrm{~kg}$. The volumes of the air-gun and the acceleration tube are $0.28 \mathrm{~m}^{3}$ and $0.142 \mathrm{~m}^{3}$. The experimental result is shown in Figure 3(a) and 3(b) where the initial pressure is the difference between the pressure of the air in the air-gun and the air environment.

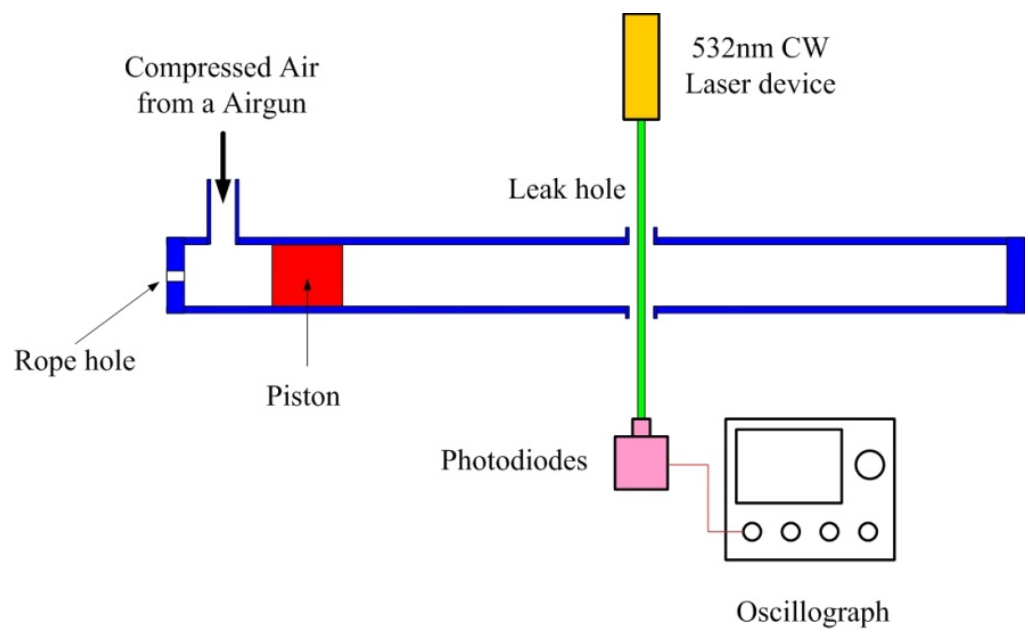

Fig. 2 Experimental setup for the piston accelerated by the compressed air and the velocity measurement

In order to study the relation of the initial pressure of the compressed air in the air-gun to the velocity and the kinetic energy of the assembly of the moving parts in the acceleration process, a formula has established between the expansion of the air and the kinetic energy of the assembly:

$$
P_{0} \cdot S \cdot L \cdot g \cdot \eta=\frac{1}{2} m V^{2}
$$

where it is assumed that the compressed air provides a stable thrust, which is equal to the initial pressure, $P_{0}$, on the piston, and the cross section and the length of the acceleration tube are $S$ and $L$, respectively. The mass and the final velocity of the assembly are $m$ and $V$, respectively. $g$ and $\eta$ are the gravity factor and the transform constant of the energy from the expansion of the air to the kinetic energy of the assembly. 

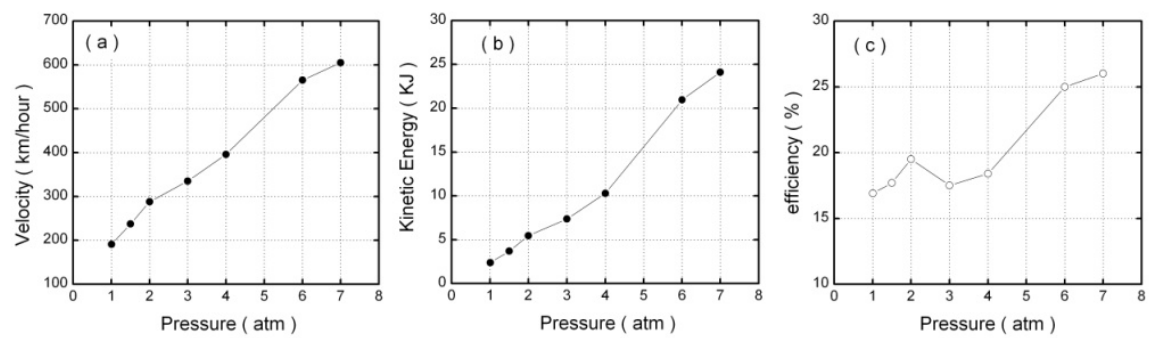

Fig. 3 Experimental results: (a) piston velocity, (b) piston kinetic energy, and efficiency of expansion to moving piston versus the initial pressure of the air in the air-gun

Figure 3 shows the experimental results for the acceleration of the piston by the compressed air. It is obvious that the efficiency of energy conversion is directly proportional to the initial pressure of the air. For a piston with a mass of $3.3 \mathrm{~kg}$, the velocity of the piston reaches $378 \mathrm{~km} /$ hour with an efficiency of $33 \%$. It is reason that the ratio of the energy loss due to the friction between the surface of the piston and the wall of the tube to the kinetic energy of the piston gradually reduce with the increasing of pressure.
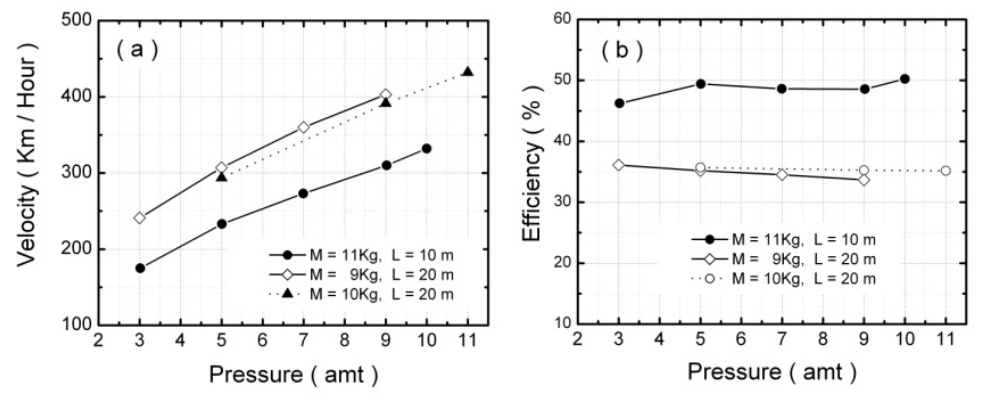

Fig. 4 (a) Velocity of model and (b) Efficiency of the energy transform from the compressed air to the kinetic energy of the device, the towrope, and the dragging device versus the initial pressure of the air in the air-gun.

In order to verify the feasibility of the acceleration with a dragging device for the operation of the moving train model testing system, the experimental setup, as shown in Figure 1(a), is designed where the diameter of the acceleration is still $110 \mathrm{~mm}$ and the drag device can slide fast on a set of double track. After the piston has passed through the leak hole, the dragging device begins to decelerate and also pull the piston backward in the deceleration tube through the towrope so that the piston also slows down. By changes in the weight of the assembly include the piston, the towrope, and the dragging device and the length of the acceleration tube, respectively, the characteristics of the energy conversion is experimentally investigated where the air gun is still with a volume of 280 liter and the experimental results are shown in Figure 4. 
It can be found that the efficiency of the energy transform from the compressed air to the moving assembly approximately maintains a constant as the length of the acceleration tube is fixed, which may be basis of the determination of the operating parameter of the testing system. On the other hand, the longer the acceleration tube, the lower the transform efficiency of the energy at the air gun with a fixed volume. It is interpreted that the pressure of the compressed air decreases with the piston moves forward in the deceleration tube and the longer the tube and the larger the volume of the air expansion. At the end of the tube, the pressure of the air decreases a special amount, which is directly proportional to the length of the tube. Therefore, in order to maintain a high efficiency, it is required that the ratio of the volume of the air gun to that of the deceleration tube. The experimental results also show that the energy conversion is improved as the weight of the moving assembly increases, as shown in Figure 4(b).

\section{Conclusions}

In summary, a project for the acceleration of the move train model by compressed air from an air gun is proposed and investigated where the air drives the piston forward and the latter pulls a dragging device. The dragging device can make the train model decelerate. In order to verify the feasibility and the corresponding operating parameters, two types of experiment are designed. The experimental results not only examine the technique but also specify the characteristics of the structure and the operation of the testing system, which can be described by a simple formula.

\section{References}

Tian, H.Q.: Train Aerodynamics, pp. 101-102. China Railway Publishing House (2007)

Zonglin, J.L., Matsuoka, K., Sasoh, A., Takayama, K.: Numerical and experimental investigation of wave dynamic processes in high-speed train/tunnels. Acta Mechanica Sinica 18(3) (2002)

Takayama, K., Sasoh, A., Onodera, O., et al.: Experimental investigation on tunnel sonic boom. Shock Waves 5, 127-138 (1995)

Sasoh, A., Matsuoka, K., Nakashio, K., et al.: Attenuation of weak shock waves along pseudo perforated walls. Shock Waves 8, 161-172 (1998)

Demmenie, E.A., de Bruin, A.C., Klaver, E.: Experimental pressure wave research at NLR for high-speed rail tunnels, NLR-TP-98375 (1998) 